\title{
High Speed Intra-Body Communication for Personal Health Care
}

\author{
Hongjie Zhu, Ruoyu Xu, and Jie Yuan \\ Department of Electronic and Computer Engineering, \\ The Hong Kong University of Science and Technology, \\ Clearwater Bay, Kowloon, Hong Kong \\ Email: \{ee_zhxaa, xuruoyu, eeyuan\}@ust.hk
}

\begin{abstract}
Intra-body communication (IBC) uses the conductive human body as the communication channel for data exchange. As the bio-sensor research is moving towards portable, wearable and implantable designs, IBC has significant potential applications for personal health care. Previous IBC research mainly focused on the electromagnetic (EM) IBC scheme, which resulted in low data rate (kbps). In this paper, we systematically studied the electric field (EF) IBC scheme, which enables 10Mbps data rate. The human body channel is thoroughly measured with attenuation, noise and distortion for the IBC application. High speed EF IBC links are tested with various modulation schemes. The minimum transmission power of different links is measured.
\end{abstract}

\section{INTRODUCTION}

$\mathrm{P}$ ERSONAL health care demands new designs of portable, wearable and implantable bio-medical sensors, as well as new methods of collecting distributed sensory information. Human body can be used as a communication medium for data exchange between sensors [1], which is normally referred to as intra-body communication (IBC). Compared with wireless body area network (BAN) which generally uses existing wireless techniques in the air channel, IBC that uses the body channel can have lower attenuation [2]. Hence, bio-medical sensors equipped with IBC transceivers can possibly consume lower power.

Previous IBC research mainly focused on transmitting low-data-rate bio-medical data, such as electrocardiogram (ECG), blood pressure (BP) and pulse oximetry (SpO2) [3]. However, much higher data rate is desirable for many new bio-medical sensors. A $64 \times 64$ implanted imaging sensor in the capsule endoscopy could generate data with $2 \mathrm{Mbps}$ data rate [4]. Wireless communication links at $\mathrm{MHz}$ are needed for neural recording [5].

The galvanic IBC scheme [6] treats the human body as a waveguide. The transmitter (TX) injects signals differentially into the human body, which generates an electro-magnetic (EM) field in the body. At the remote end, the receiver (RX) electrodes sense the EM field. Nonetheless, the data rate in this scheme is generally low in the kbps range because the body effectively shorts the TX electrodes, which enhances the channel attenuation. Electric field (EF) IBC transmits the signal through the body with an open return path [1], which reduces the channel attenuation so that it enables much higher data rate than the EM IBC [7]. So far, the study on EF IBC remains incomplete. Important properties of communication channels, such as noise and distortion, have never been characterized.

In this work, the EF IBC channel is systematically investigated. The body channel attenuation, noise, and distortion are thoroughly measured and analyzed. 10Mbps EF IBC links are established over the body with common modulation schemes of BPSK, QPSK, and 16QAM. The minimum transmission power of the links is measured.

\section{THE EF IBC SYSTEM}

\section{A. EF IBC System Modeling}

An EF IBC system includes a transmitter (TX), a receiver (RX), and the EF IBC channel which consists of both the conductive human body forward path and the parasitic return path. In EF IBC, both TX and RX connect one electrode on the body, while leaving the other electrode floating [1]. The floating electrodes are parasitically coupled to the ground to close the circuit loop. In a typical application in Fig. 1, the TX is built in a bio-medical sensor, and sends signals into the IBC channel. The RX built in a receiving device, such as a wrist watch, can collect signals from TXs of different bio-medical sensors and deliver the sensory data to medical service centers through public $3 \mathrm{G}$ wireless network.

Similar to other communication channels, the EF IBC channel can be characterized by the attenuation profile, the noise and distortion level as shown in Fig. 2. The channel attenuation generally varies with frequency. The low-loss windows are desirable communication bands. On the other hand, this frequency dependent attenuation distorts the signal. In digital communication, this channel distortion causes previous symbols interfering the subsequent symbols, which 


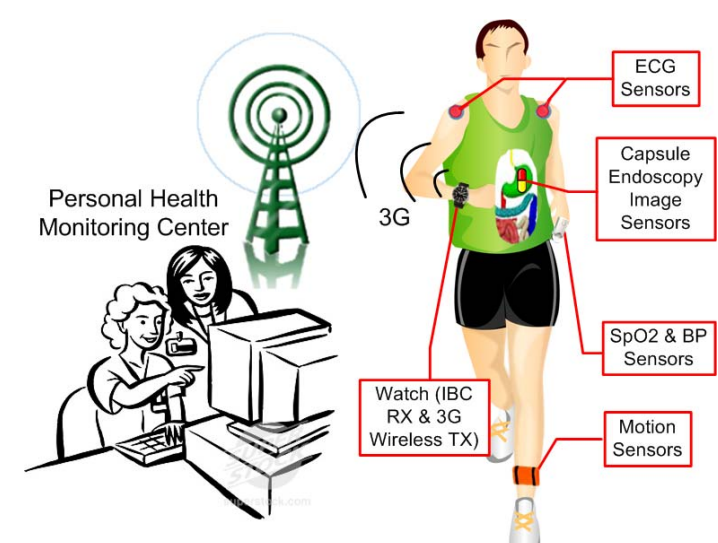

Fig. 1. A typical EF IBC application: Personal health information collected by the bio-medical sensors is sent to the IBC channel. The IBC RX built-in the watch receives the data and the watch send them to the health monitoring center through $3 \mathrm{G}$ wireless network.

is generally known as the inter-symbol interference (ISI) [8].

\section{B. Digital Modulation}

For EF IBC applications, the EF IBC channel is not band-limited. Three common digital modulation schemes are tested over the channel, which are BPSK, QPSK, and 16QAM. Bit error rate (BER) is an important parameter for data communication in low-power links [8]. In an additive white Gaussian noise (AWGN) channel, BER for BPSK, QPSK and 16QAM can be calculated from the received signal-to-noise ratio (SNR) with Eqn. 1, Eqn. 2 and Eqn. 3, respectively [8].

$$
\begin{aligned}
& B E R_{B P S K}=Q\left(\sqrt{\frac{E_{b}}{N_{0} / 2}}\right)=Q\left(S N R_{S y m}\right) \\
& B E R_{Q P S K}=Q\left(\sqrt{\frac{E_{S y m} / 2}{N_{0} / 2}}\right)=Q\left(\frac{\sqrt{2}}{2} S N R_{S y m}\right) \\
& B E R_{16 Q A M}=3 Q\left(\sqrt{\frac{1}{10} \cdot \frac{E_{a v}}{N_{0} / 2}}\right)=3 Q\left(\frac{1}{\sqrt{10}} S N R_{S y m}\right)
\end{aligned}
$$

where $E_{b}$ is the bit energy. $E_{\text {Sym }}$ is the symbol energy. $E_{a v}$ is the average symbol energy. $\mathrm{N}_{0} / 2$ is the noise density.

\section{MEASUREMENT SETUPS}

Fig. 3 shows the measurement setup for attenuation characterization. A Rhode \& Schwarz ZVB8 vector network analyzer (VNA) is used to measure the s-parameters of the EF IBC system. The $\mathrm{S}_{21}$ indicates the body channel attenuation. Because shields of every VNA port are connected inside the equipment, the parasitic return path in the EF IBC channel will be shorted if both TX and RX are directly connected to the VNA ports. In order to keep the floating return path as it is in the real EF IBC systems, baluns (FTB-1-1 A15 from Mini-Circuits) are used to separate the VNA ports from TX and RX. The PCB board is shown in Fig. 3(b). The copper

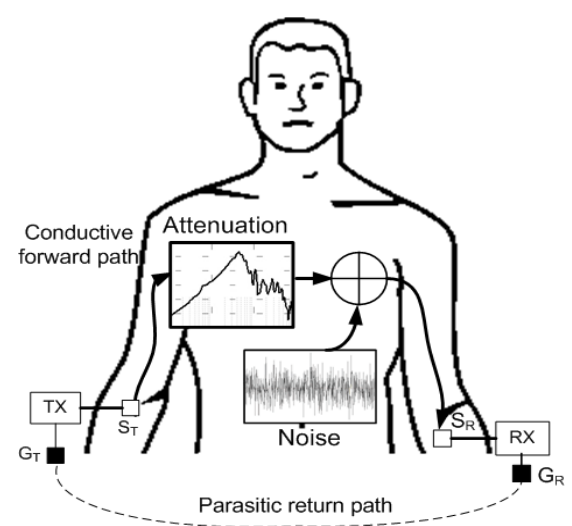

Fig. 2. Block diagram of the EF IBC channel model: The EF IBC channel can be characterized by the attenuation profile, the noise and distortion level in the channel.

signal electrode is soldered to the microstrip on the front side of the board, which is connected to the center of an SMA connector. The shield of the SMA connector is soldered to the ground plane on the back side of the board. The noise power spectrum density (PSD) in the EF IBC channel is measured by an Agilent E4404B ESA-E series spectrum analyzer (SA) with zero transmission power.

Fig. 4 shows digital communication setup over the EF IBC channel. A Rhode \& Schwarz vector signal generator (VSG) SMU200A is used to generate modulated signals for BPSK, QPSK and 16QAM at different symbol rates with a center frequency which is the frequency for the lowest channel attenuation. An Agilent Infiniium DSA90604A oscilloscope (OSC) is used to capture and analyze the signals at the RX. The received signals are demodulated and analyzed by the Agilent 89600 series vector signal analysis (VSA) software.

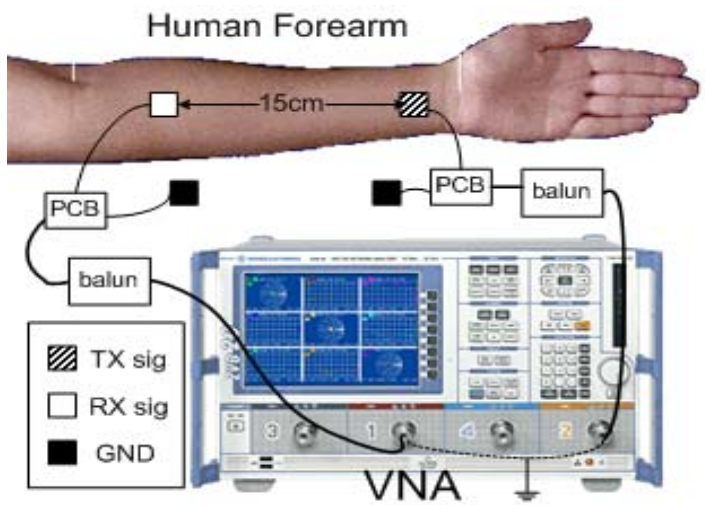

(a)

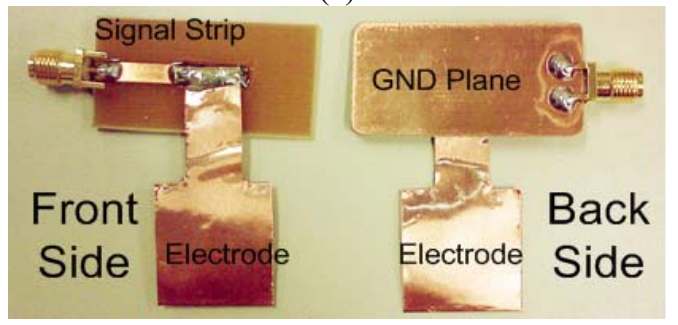

(b)

Fig. 3. (a) the measurement setup, port 2 of VNA is TX and port 1 is $\mathrm{RX}$, baluns are used to separate the GND of TX and RX; and (b) the picture of TX and RX PCB boards, the copper-foil-made electrode is soldered to the micro-strip which has characteristic impedance of $50 \Omega$. 


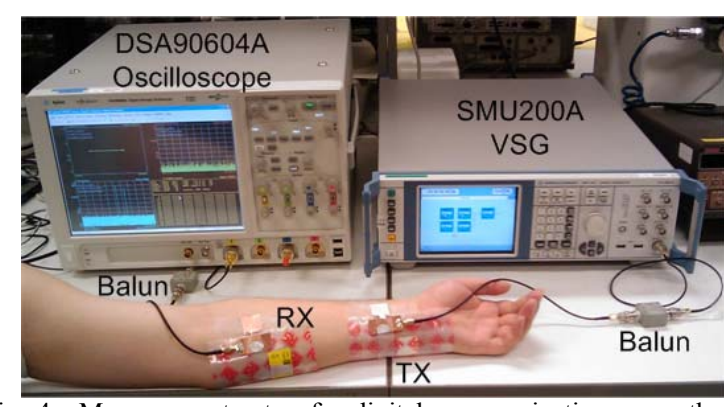

Fig. 4. Measurement setup for digital communications over the EF IBC channel: VSG is used as TX and OSC is used as RX, baluns are used to separate the GND of RX and RX.

\section{EF IBC CHARACTERIZATION RESULTS}

\section{A. Attenuation}

Fig. 5(a) shows the measured channel attenuation. The "without balun" curve is measured by connecting the TX and RX ground electrodes together, which happens when the boards are connected to the equipment directly without baluns. The IBC channel shows a low-pass profile. Similar EF IBC characteristics have been obtained in [2], which is different from the real EF IBC system.

Other curves in Fig. 5(a) are measured by the setup in Fig. 3(a) with different distances between the electrodes. The EF IBC channel generally shows a band-pass profile with a center frequency at about $42 \mathrm{MHz}$. The lowest attenuation is about $-17 \mathrm{~dB}$. Due to the capacitive coupling between the electrode and the muscle, the EF IBC channel attenuation rises with frequency at low frequency. The capacitance between the two electrodes on a PCB board increases the attenuation at high frequency, which forms the band-pass profile. A more detailed analysis of the EF IBC channel attenuation can be found in [9]. The channel attenuation at low frequency increases with distance. But the profile does not change, which indicates that the impedance of the human body is relatively flat over the spectrum.

\section{B. Distortion and Noise}

The measured noise PSD shows that the noise in EF IBC channel is almost white from $10 \mathrm{MHz}$ to $100 \mathrm{MHz}$. The received channel SNR is affected by the noise as well as the distortion. Fig. 5(b) shows the measured total channel distortion and noise power of BPSK signals with different received signal power $P_{R X}$ and different symbol rate. The total channel distortion and noise power $\mathrm{P}_{\mathrm{d}+\mathrm{n}}$ within the bandwidth of the modulated signal can be calculated by

$$
P_{d+n}=P_{R X}-S N R_{S y m}
$$

where $\mathrm{SNR}_{\mathrm{Sym}}$ is the SNR per symbol. Curves in Fig. 5(b) show $\mathrm{P}_{\mathrm{d}+\mathrm{n}}$ of the EF IBC channel is dependent on received signal if the signal is large. This indicates the channel is likely dominated by the signal-dependent ISI distortion when $\mathrm{P}_{\mathrm{RX}}$ is larger than $-30 \mathrm{dBm}$, which is due to the non-flat channel attenuation. For small signal power, $\mathrm{P}_{\mathrm{d}+\mathrm{n}}$ are almost identical, which indicates the channel is dominated by the signal-independent noise when $\mathrm{P}_{\mathrm{RX}}$ is less than $-30 \mathrm{dBm}$.

Fig. 5(c) shows the total channel distortion and noise power of the three modulation schemes at different symbol rates when the received signal power is $-30 \mathrm{dBm}$. It is worth noticing that for each curve, $\mathrm{P}_{\mathrm{d}+\mathrm{n}}$ increases about $3 \mathrm{~dB}$ when the symbol rate doubles, which doubles the signal bandwidth. This further verifies that the channel noise is approximately white in the $30 \mathrm{MHz}$ band centered at $42 \mathrm{MHz}$.

Fig. 6 (a), (b) and (c) show the constellation diagrams of received BPSK, QPSK and 16QAM signals, respectively, with symbol rate $\mathrm{S}=10 \mathrm{MSps}$ and received signal power $P_{R X}=-30 \mathrm{dBm}$. Analysis shows that the constellation points in every constellation diagram in Fig. 6 generally follow the Gaussian distribution. This indicates that the distribution of the channel noise is Gaussian.

In summary, when $\mathrm{P}_{\mathrm{RX}}$ is less than $-30 \mathrm{dBm}$, the EF IBC channel is dominated by the channel noise, which appears to be white and Gaussian. Therefore, the EF IBC channel can be considered as an AWGN channel when $\mathrm{P}_{\mathrm{RX}}$ is less than $-30 \mathrm{dBm}$.

\section{Minimum Transmission Power}

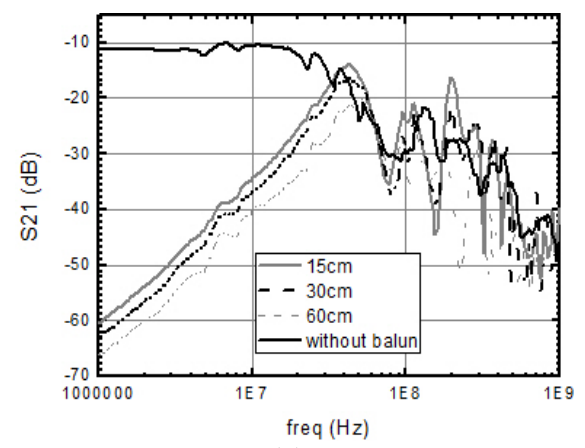

(a)

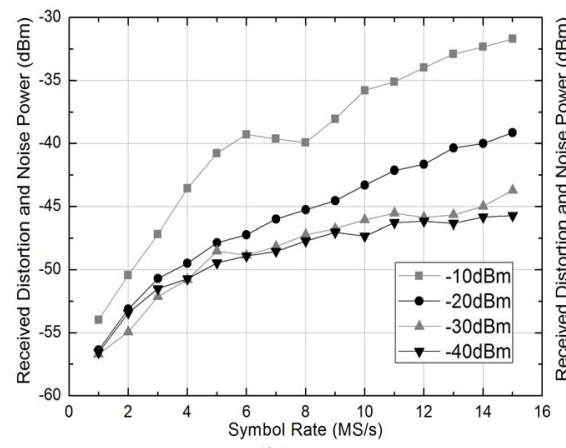

(b)

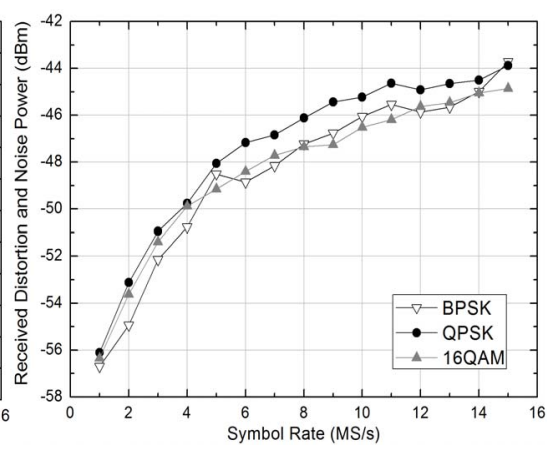

(c)

Fig. 5. (a) $\mathrm{S}_{21}$ parameter for the EF IBC measurement setup with balun and without balun, the curves use the averaged data from 45 sets of measurement results (b) the channel distortion and noise power for BPSK at different symbol rates with received signal power $P_{\mathrm{RX}}$ at $-10 \mathrm{dBm},-20 \mathrm{dBm},-30 \mathrm{dBm}$ and $-40 \mathrm{dBm}$; (c) the channel distortion and noise power at different symbol rates with received signal power $P_{\mathrm{Rx}}$ of $-30 \mathrm{dBm}$ for BPSK. OPSK and $16 \mathrm{OAM}$ 


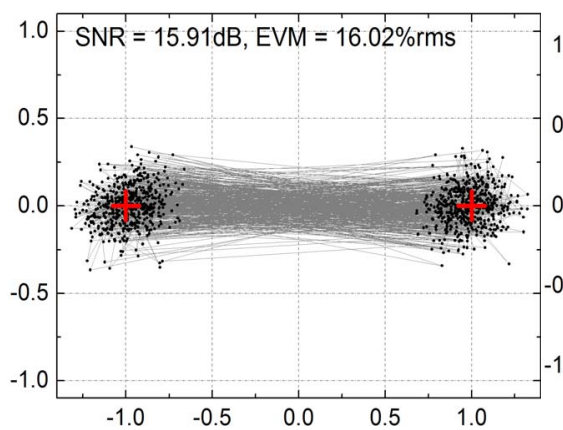

(a)

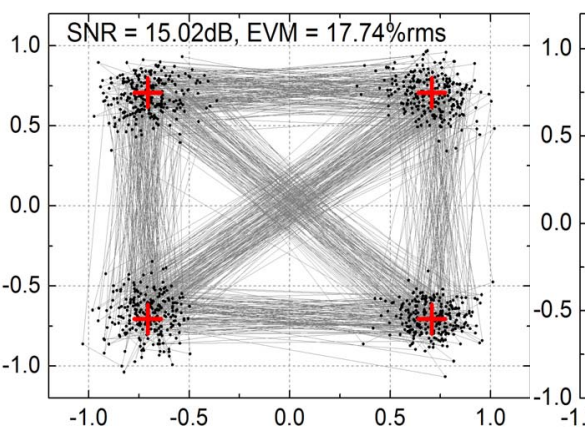

(b)

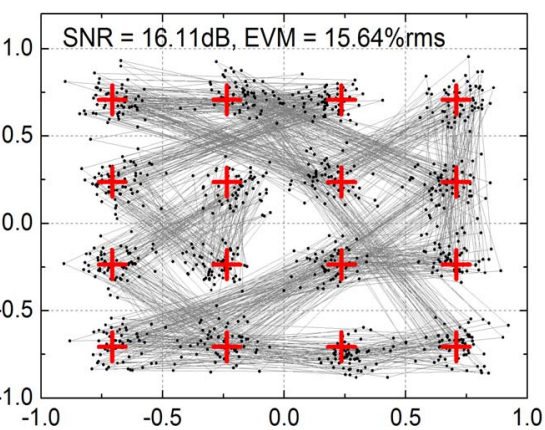

(c)

Fig. 6. Constellation diagrams for (a) BPSK at $P_{\mathrm{RX}}=-30 \mathrm{dBm}$, (b) QPSK at $\mathrm{P}_{\mathrm{RX}}=-30 \mathrm{dBm}$ and (c) $16 \mathrm{QAM}$ at $\mathrm{P}_{\mathrm{RX}}=-30 \mathrm{dBm}$, with symbol rate $\mathrm{S}=10 \mathrm{MSps}$

The minimum TX and RX power of every modulation scheme for $10 \mathrm{Mbps}$ EF IBC links with $\mathrm{BER}=10^{-3}$ and $10^{-6}$ are measured and listed in Table I and Table II, respectively. The RX power in each case is smaller than or around $-30 \mathrm{dBm}$. Therefore, EF IBC channels in these measurements are AWGN channels which validate Eqn. 1-3. To transmit data at $10 \mathrm{Mbps}$ over the EF IBC channel, the required minimum transmission power for BPSK, QPSK and 16QAM are $-21.5 \mathrm{dBm},-22.2 \mathrm{dBm}$ and $-14.0 \mathrm{dBm}$, respectively, to achieve $10^{-3} \mathrm{BER}$, and $-16.8 \mathrm{dBm},-17.8 \mathrm{dBm}$ and $-10.3 \mathrm{dBm}$, respectively, to achieve $10^{-6} \mathrm{BER}$. The required minimum transmission power of the EF IBC is between $0.5 \%$ and $10 \%$ of the transmit power of a class 3 Bluetooth radio [10].

\section{CONCLUSION}

New portable, wearable and implantable bio-medical sensors for personal health care demand low-power and high-speed communication techniques for data exchange. Unlike previous WBAN techniques and the EM IBC techniques, the EF IBC technique is able to meet both demands well.

In this work, an effective EF IBC characterization setup is developed and the body channel characteristics are measured. Measurement results show the EF IBC channel has a

TABLE I

THE REQUiRED MINIMUM TX AND RX POWER FOR BER $=10^{-3}$

\begin{tabular}{cccc}
\hline \hline $\begin{array}{c}\text { Modulation } \\
\text { Scheme }\end{array}$ & $\mathrm{SNR}(\mathrm{dB})$ & $\mathrm{P}_{\mathrm{RX}}(\mathrm{dBm})$ & $\mathrm{P}_{\mathrm{TX}}(\mathrm{dBm})$ \\
\hline BPSK & 9.80 & -35.5 & -21.5 \\
QPSK & 12.81 & -35.9 & -22.2 \\
$16 Q A M$ & 20.64 & -28.7 & -14.0 \\
\hline \hline
\end{tabular}

For each modulation scheme: $\mathrm{BER}=10^{-3}$, Bit Rate $=10 \mathrm{Mbps}$

TABLE II

THE REQUiRED Minimum TX AND RX POWER FOR BER $=10^{-6}$

\begin{tabular}{cccc}
\hline \hline $\begin{array}{c}\text { Modulation } \\
\text { Scheme }\end{array}$ & $\mathrm{SNR}(\mathrm{dB})$ & $\mathrm{P}_{\mathrm{RX}}(\mathrm{dBm})$ & $\mathrm{P}_{\mathrm{TX}}(\mathrm{dBm})$ \\
\hline BPSK & 13.54 & -30.3 & -16.8 \\
QPSK & 16.55 & -32.2 & -17.8 \\
$16 Q A M$ & 23.93 & -25.4 & -10.3 \\
\hline \hline
\end{tabular}

For each modulation scheme: $\mathrm{BER}=10^{-6}$, Bit Rate $=10 \mathrm{Mbps}$ band-pass profile attenuation with the low-loss window around 42MHz. High speed communication links are tested over the EF IBC channel. The channel SNR is affected by both the signal-dependent distortion and the signalindependent noise. When the received signal power is larger than $-30 \mathrm{dBm}$, the distortion dominates the channel. When the received signal power is less than $-30 \mathrm{dBm}$, the channel is dominated by noise. The channel noise appears to be white and Gaussian. For 10Mbps EF IBC links with $10^{-3}$ BER, the minimum transmission power for BPSK, QPSK and 16QAM are $-21.5 \mathrm{dBm},-22.2 \mathrm{dBm}$ and $-14.0 \mathrm{dBm}$, respectively. For $10 \mathrm{Mbps}$ EF IBC links with $10^{-6} \mathrm{BER}$, the minimum transmission power are $-16.8 \mathrm{dBm},-17.8 \mathrm{dBm}$ and $-10.3 \mathrm{dBm}$, respectively.

\section{REFERENCES}

[1] T.G. Zimmerman, "Personal Area Networks: Near-Field intrabody communication”, IBM System Journal, Vol. 35, pp. 609-617, NOS 3\&4, 1996.

[2] J. A. Ruiz, Jiang Xu and S. Shimamoto, "Propagation characteristics of intra-body communications for body area networks," Consumer Communications and Networking Conference, 2006. CCNC 2006. 3rd IEEE, vol. 1, pp. 509-513, 2006.

[3] M. S. Wegmueller, W. Fichtner, M. Oberle and N. Kuster, "BPSK \& QPSK Modulated Data Communication for Biomedical Monitoring Sensor Network," Engineering in Medicine and Biology Society, 2006. EMBS '06. 28th Annual International Conference of the IEEE, pp. 2071-2074, 2006.

[4] H. Chan, and J. Yuan, "Current-Mode Temporal Difference CMOS Imager for Capsule Endoscopy", 2007 IEEE Biomedical Circuits and Systems Conference(BioCAS), pp. 219-222, Montreal, Canada, Nov. 27-30, 2007.

[5] C. Chestek, P. Samsukha, M. Tabib-Azar, R. Harrison, H. Chiel, and S. Garverick, "Microcontroller-based wireless recording unit for neurodynamic studies in saltwater", IEEE Sensors J., Vo. 6, pp. 1105-1114, Oct. 2006.

[6] M. S. Wegmueller, A. Kuhn, J. Froehlich, M. Oberle, N. Felber, N. Kuster and W. Fichtner, "An Attempt to Model the Human Body as a Communication Channel," Biomedical Engineering, IEEE Transactions on, vol. 54, pp. 1851-1857, 2007.

[7] K. Hachisuka, Y. Terauchi, Y. Kishi, T. Hirota, K. Sasaki, H. Hosaka and K. Ito, "Simplified circuit modeling and fabrication of intrabody communication devices," Solid-State Sensors, Actuators and Microsystems, 2005. Digest of Technical Papers. TRANSDUCERS '05. the 13th International Conference on, vol. 1, pp. 461-464 Vol. 1, 2005.

[8] J. Proakis, "Digital Communications", $4^{\text {th }}$ edition, McGraw-Hill, 2001.

[9] R. Xu, H. Zhu, J. Yuan, "Characterization and Analysis of Intra-body Communication Channel”, 2009 IEEE International Symposium on Antennas and Propagation (APS).

[10] Bluetooth Core Specification v3.0+HS, [Online] http://www.bluetooth. $\mathrm{com} /$ Bluetooth/Technology/Building/Specifications/Default $/ \mathrm{htm}$ 